\title{
近场微波显微镜在超导材料高通量表征中的 应用与前景
}

\author{
秦明阳，石玉君，魏忠旭，朱北沂，袁洁"，金鬼* \\ 中国科学院物理研究所, 北京凝聚态国家实验室, 北京 100190 \\ *联系人, E-mail: yuanjie@iphy.ac.cn; kuijin@iphy.ac.cn
}

2017-06-30 收稿, 2017-07-18 修回, 2017-07-18 接受, 2017-10-12 网络版发表

国家重点研发计划(2015CB921000, 2016YFA0300301, 2017YFA0303003)、中国科学院前沿科学重点研究项目(QYZDB-SSW-SLH008, QYZDYSSW-SLH001)、国家自然科学基金(11674374, 11474338)、北京市科技计划(Z161100002116011, D161100002416003, D161100002416001)和 中国科学院战略性先导科技专项(B 类)(XDB07020100, XPDB01-01)资助

\begin{abstract}
摘要 “材料基因组计划” 旨在缩短材料的研发周期, 作为材料研发中不可或缺的实验环节, 各种高通量合成和 快速表征技术的发展也越来越受到重视。近场微波显微镜因具有微区扫描快速表征的能力，适用于组合材料的微 波特性快速表征. 本文针对超导材料的一些重要物理参量, 分析了近场微波显微镜对高通量薄膜快速表征的个别 案例. 并以衰逝微波探针显微镜为例，介绍了该类仪器的构成、测量原理和数据分析方法，简要探讨了其应用于超 导机理和电子学器件研究的前景.
\end{abstract}

关键词＼cjkstart材料基因组，高通量实验技术，组合薄膜，近场微波显微镜

高温超导材料在电力传输、磁悬浮、储能、核磁 共振成像等诸多方面都有极其重要的应用前景, 因 此寻找具有更高转变温度 $\left(T_{\mathrm{c}}\right)$ 的超导材料是一项具有 重要意义的课题 ${ }^{[1]}$. 相对于单质超导体不超过 $10 \mathrm{~K}$ 的 超导转变温度, 目前报道的常压下 $T_{\mathrm{c}}$ 记录 $(138 \mathrm{~K})$ 由 $\mathrm{Hg}_{0.8} \mathrm{Tl}_{0.2} \mathrm{Ba}_{2} \mathrm{Ca}_{2} \mathrm{Cu}_{3} \mathrm{O}_{8.33}$ 保持, 其构成元素多达6种 ${ }^{[1]}$, 这自然启发大家到多元化合物材料中去寻找更高 $T_{\mathrm{c}}$ 的超导体. 除了元素的数目增加以外, 高温超导电性 还对化合物中各元素配比异常敏感. 如在214结构的 铜氧化物超导体(如 $\mathrm{La}_{2} \mathrm{CuO}_{4}$ ) 中, $1 \%$ 的阳离子掺杂浓 度改变会导致材料在绝缘-超导-金属行为之间的转 变 ${ }^{[2]}$, 而氧含量的细微差别也会引起氧化物超导体性 能的显著变化 ${ }^{[3]}$, 因此寻找新的超导体需要建立完 整、精确的材料相图. 多元素排列组合、多参量调控 意味着新型超导材料的探索不可避免地会遇到从庞
大数量的样品库中篮选合适材料的难题. 传统的材 料研究采用的是“单一条件(组分)合成-测试-调整参 数-再合成”的“顺序迭代”试错模式，这种模式是行之 有效的，但是随着材料体系的越发复杂和人们对于 高综合性能材料需求的日趋紧迫，简单的“顺序迭 代”试错法已经难以满足现代新材料研发中高效和低 成本的需求, 这需要人们尝试打造全新的研发流程, 加速先进材料的发现与应用. 在此背景下，材料基因 组计划 (materials genome initiative, MGI) ${ }^{[4]}$ 被提出.

材料基因组计划是包含材料计算与大数据处理、 高通量材料制备与表征、高通量技术研发三大主要功 能的、以材料探索、基础与应用研究为目标的重要战 略性课题. 其中, 高通量计算结合大数据等信息化技 术手段, 开展智能化材料设计和调控、物性计算与模 拟，能够做到在短时间内对大量材料进行预估，为实

引用格式: 秦明阳, 石玉君, 魏忠旭, 等. 近场微波显微镜在超导材料高通量表征中的应用与前景. 科学通报, 2017, 62: 4025-4036 Qin M Y, Shi Y J, Wei Z X, et al. Applications and perspective of near-field microwave microscope in high-throughput characterizations of superconducting materials (in Chinese). Chin Sci Bull, 2017, 62: 4025-4036, doi: 10.1360/N972017-00722 
验制备提供理论指导, 提高新材料的发现几率 ${ }^{[5]}$. 高 通量材料制备技术能连续地调控某个实验参数如温

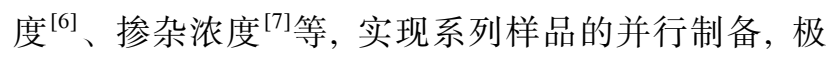
大地缩短样品的合成周期. 包含微区集成、连续扫 描、多功能叠加等手段在内的高通量表征技术具有高 时空分辨率, 能高速、瞬态地对样品进行原位的结 构、成分和各种物理属性的表征, 可大幅度提高多元 材料物性探索的效率 ${ }^{[8]}$. 但快速表征手段一般不具有 通用性, 针对具体材料需定制高通量表征技术. 高通 量技术研发致力于不断发展新方法与新技术, 实现 关键实验设备的突破. 材料基因组计划的实施能有 效缩短新材料的研发周期, 大幅降低成本. 一旦确定 所需材料的具体组分, 便可进一步精细优化材料制 备条件并获得高质量样品, 推动相关基础研究的同 时也为材料的推广与应用奠定坚实的基础.

\section{1 高通量材料制备与表征技术}

作为 MGI三大要素之一, 高通量材料制备与表 征技术的核心思想是将传统材料研究中采用的“顺序 迭代”改为 “并行处理”. 它与 “材料计算与大数据处 理” 和 “材料信息学与数据库” 紧密结合, 在由传统经 验方法向新型预测方法的过渡中, 扮演着承上启下 的角色, 可以加速材料的篮选和优化 ${ }^{[4]}$.

\section{1 组合薄膜制备技术}

在现有的高通量材料制备技术中, 组合薄膜技 术最为成功, 应用也相对广泛. 组合薄膜技术的发展 大致分为 3 个阶段 ${ }^{[8]}$ : 第一代组合薄膜的出现可以追 溯到 1965 年, Kennedy等人 ${ }^{[9]}$ 利用电子束同时蒸发 Fe, $\mathrm{Cr}, \mathrm{Ni} 3$ 个源, 在一块边长为 $10 \mathrm{in}$ 的等边三角形金属 䈃片上获得了 $\mathrm{Fe}-\mathrm{Cr}-\mathrm{Ni}$ 三元体系相图. 第二代组合薄 膜出现于 20 世纪 90 年代, Xiang等人 ${ }^{[10]}$ 利用阵列式窗 口掩膜来控制前驱物在衬底上的分布, 经过20步沉 积和后退火处理, 在仅 $2.5 \mathrm{~cm} \times 2.5 \mathrm{~cm}$ 的 $\mathrm{MgO}$ 或 $\mathrm{LaAlO}_{3}$ 基片上合成包含有 1024 种组分的铜基氧化物 薄膜, 其中包含了 $\mathrm{Bi}$ 系、Y系等多种已知的超导组分, 该工作为利用组合薄膜技术快速合成多种超导体开 启了大门. 这项技术因所合成的材料类似基因芯片 被称为组合材料芯片技术. 为了能够获得单晶品质 的梯度组分薄膜, 先进的激光分子束外延 (laser molecular beam epitaxy, LMBE)生长法被融入至组合制 备技术, 诞生了组合激光分子束外延生长技术, 即第
三代组合薄膜生长技术 ${ }^{[7]}$. 它在利用掩膜的同时, 还 利用反射高能电子衍射仪, 实时监控样品的生长过 程, 控制不同的前驱成分在一个原胞层内进行组合, 实现样品的逐层生长, 从而确保样品的质量.

第三代组合薄膜生长技术的原理如下：通过激 光(以短波长脉冲分子激光为主)与靶材 (化合物粉末 压制)相互作用，靶材表面形成的等离子体扩散至衬 底, 沉积形成所需的薄膜样品. LMBE腔体处于高真 空, 这保证了薄膜的外延生长. 如在材料沉积过程 中, 利用随时间移动的掩膜板使成分 1 在基片表面形 成沉积率梯度分布, 然后反方向移动掩膜板使成分 2 形成反方向的沉积率梯度分布, 确保这两种成分在 一个原胞的尺度内混合, 即形成梯度组分薄膜, 如图 1 所示. 该方法可获得连续线性梯度的成分分布, 适 用于高精度的材料相图研究 ${ }^{[4]}$.

\section{2 高通量快速表征技术}

高通量快速表征技术不仅可以进行材料快速篮 选, 还能提供样品表征数据, 快速建立样品性能与各 种参数的依赖关系, 从而给出样品优化的线索 ${ }^{[1]}$. 下 面介绍几种高通量的快速表征技术.

高通量微区结构表征. 对材料结构的表征通常 采用XRD(X射线衍射), 该方法可以检测样品成相质 量. XRD图谱包含了晶体结构的大量信息, 高分辨 XRD结合微区扫描组件便可对薄膜样品进行准一维 或准二维测量, 从而实现对高通量组合薄膜结构的 快速表征 ${ }^{[12]}$.

高通量微区光学性质表征. 光学表征技术常用 于分析材料的禁带宽度, 椭偏仪是一种光学技术分 析和计量仪器, 可以测量薄膜的光学常数和厚度. 除 此之外，成像椭偏仪结合自动消光椭偏技术和传统 显微技术, 还可实现超薄膜的实时可视化分析测试,

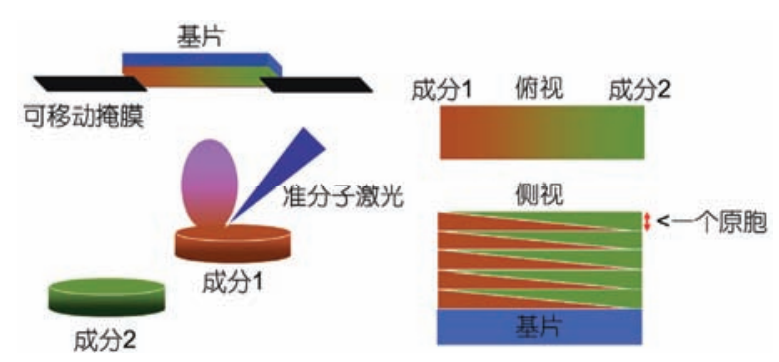

图 1 (网络版彩色)第三代组合薄膜制备方法

Figure 1 (Color online) The third-generation combinatorial film fabrication method 
能够实时看到样品微观尺度上的结构, 快速测定高 温超导、巨磁电阻、多铁和拓扑绝缘体等薄膜材料的 光学参量.

高通量微区热学性能表征. 材料的热学性质关 系到很多功能材料的使用效率, 同时也是固体材料 内部晶格振动过程的最直接反映. 激光热学表征技 术使得微区热学性质测量成为可能, 在表征系统中 通过脉冲激光加热和激光迈克尔逊干涉效应, 可精 密测量固体材料局域热扩散、热传导系数、热膨胀系 数. 该方法对形貌要求不高, 可测量块体和薄膜材料, 测量时间短, 因此可以对高通量材料进行快速篮选.

除上述几种方法之外, 高通量表征技术还包括 高通量微区电磁学、电化学、力学、磁学、催化性能 测量和原位、跨尺度统计分布表征技术. 相对于已经 广泛应用的组合薄膜制备技术, 针对材料物性的高 通量快速表征手段仍需要进一步地发展和开拓, 以 提升整个材料基因组计划中高通量实验环节的效率. 从已有的技术手段来看, 一种测量方法能够用于组 合薄膜的快速表征, 需具备两个基本特征: (1) 具备 有空间分辨能力的“探针”, 这里的“探针”是广义上的 “针”, 既包括原子力显微镜 (atomic force microscope, AFM)、扫描隧道显微镜 (scanning tunneling microscope, STM) 中的实物针尖, 也包括磁光克尔显微镜、 扫描电子显微镜中由 “透镜”汇聚形成的场或束流的 针尖; (2) “探针”能够在样品表面快速扫描, 这里的 扫描既可以是机械式的扫描, 也可以是由外场控制 的偏转. 选择合适的探针和扫描模式, 便可以实现对 组合薄膜各种物理特性的高通量表征.

材料的微波特性是当今功能材料备受关注的性 质之一, 对于材料在高频领域的应用尤为重要. 扫描 近场微波显微镜 (near-field microwave microscope, $\mathrm{NMM}$ )作为一种具备高空间分辨能力的表征设备, 可 用于各类材料介电常数、电导率等基本电磁学特性的 高通量表征. 下面以超导材料为例, 简单介绍近场微 波显微镜测量穿透深度和介电常数等参数的基本原 理, 讨论其在超导配对对称性研究、超导微波器件品 质因数优化等方面的前景.

\section{2 超导微波表征与近场微波显微镜}

微波因其特定波段 $(1 \mathrm{~mm}$ 1 m) 和准静态特征而 具有穿透能力强、易定量分析等优势, 这使得它常被 用于表征超导体的表面阻抗. 近年来 $\mathrm{MGI}^{[4]}$ 和纳米科
学 ${ }^{[13]}$ 的兴起对样品表征手段提出了快速、无损、定 量、高分辨等新要求, 而近场微波显微镜恰能满足此 需要.

继 1911年Onnes ${ }^{[14]}$ 发现超导的零电阻特性之后, Meissner等人 ${ }^{[15]}$ 于 1933 年又发现了超导的第二个特 性一一完全抗磁性. 1935年, London兄弟 ${ }^{[16]}$ 提出了两 个电磁学方程对此给予了唯象解释: 当材料处于超 导态时, 磁场会因屏蔽而在样品内呈现指数衰减, 其 中衰减的特征长度 $\lambda_{\mathrm{L}}=\sqrt{m /\left(\mu_{0} n_{s} e^{2}\right)}$, 被称作穿透深 度. 穿透深度与库柏对密度密切相关 ${ }^{[17]}$, 可以帮助 我们深人了解超导的能隙特征与配对对称性 ${ }^{[18]}$, 是 超导体的重要特征参量之一.

目前, 测量高温超导穿透深度的方法主要分为 以下 3 类: 直流、交流磁化率和高频动态电感法; 微 波表面阻抗法和微带谐振技术; $\mu$ 子自旋共振、电子 顺磁共振和极化中子反射技术 ${ }^{[19]}$. 其中微波表面阻 抗法因理论直观、测量方便而被广泛应用，它以趋肤 效应和二流体模型为基础，给出 $T_{\mathrm{c}}$ 以下超导材料表 面电抗的变化量 $\Delta X_{s}$ 与穿透深度的变化量 $\Delta \lambda$ 存在关

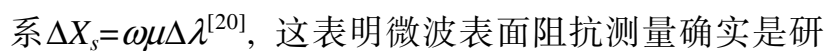
究超导穿透深度的有效方法. 1989年, Drabeck等人 ${ }^{[21]}$ 通过测量不同衬底上 $\mathrm{YBa}_{2} \mathrm{Cu}_{3} \mathrm{O}_{7}$ 样品的微波表面阻 抗发现该材料的微观机制与 BCS 理论吻合. $\mathrm{Wu}$ 等 人 $^{[22]}$ 于 1993 年利用微波谐振腔测得电子型铜氧化物 超导体 $\mathrm{Nd}_{1.85} \mathrm{Ce}_{0.15} \mathrm{CuO}_{4}$ 穿透深度的温度依赖关系, 给出其为单能隙 $\mathrm{S}$ 波配对的 $\mathrm{BCS}$ 超导体. Hardy等人 ${ }^{[23]}$ 也利用微波技术研究了超导单晶样品 $\mathrm{YBa}_{2} \mathrm{Cu}_{3} \mathrm{O}_{6.95}$ 穿 透深度的低温特性, 支持其序参量的 $d_{x^{2}-y^{2}}$ 对称 性 $^{[24 ~ 26]}$.

然而在如上所述的传统微波实验中, 样品与源 的距离都远大于波长，也就是说测量的是 “远场”信 号. 这将导致较多局限性 ${ }^{[27]}$ : 一方面, 仪器工作在远 场尺度下, 所测得的将是样品各个区域响应的积分, 因此难以获得材料在纳米尺度下的微区特性, 尤其 是对于组合薄膜材料, 远场测试都将失效; 另一方 面, 材料边角处大屏蔽电流的存在使测量结果在很 大程度上依赖于材料的几何外形，这会造成实验分 析的困难.

然而, 探针与样品在“近场”范围内的相互作用 恰恰可以打破以上限制. 为界定探针与样品间的相 互作用属远场还是近场, 需引人参量一一探针的特征 尺寸 $D$. 根据 $D$ 与光源波长 $\lambda$ 、探针与样品间矢量半径 
$\boldsymbol{r}$ 之间的关系, 可将相互作用范围划分为近场、中间 场和远场 ${ }^{[27]}$. 在远场 $\left(\begin{array}{lll}D & \lambda & r\end{array}\right)$, 探针向外辐射波 矢 $k=\omega / \mathrm{c}$ 的行波, 且电磁场的幅度以 $1 / r$ 的形式衰减, 相位在垂直 $r$ 的方向上相同; 在近场 $\left(\begin{array}{ll}D \leqslant r & \lambda\end{array}\right)$, 电 磁场具有静态特征且非常依赖于探针形状, 其幅度 以 $1 / r^{2}$ 或更快的速度衰减, 其中与 $r$ 有纯指数依赖的 近场电磁波叫做衰逝波, 此时电磁场的波矢 $k \sim 1 / D$ $(\text { 空间分辨率约为 } D)^{[28,29]}$.

1928 年, Synge ${ }^{[30]}$ 最先提出突破分辨率极限 $(\lambda / 2)$ 的想法. 他设想在一个不透明的屏上开一直径小于 光源波长的圆孔作为点源, 将它置于平整透明样品 表面上方约 $10 \mathrm{~nm}$ 的位置, 再利用此点源发射探测光 对样品进行扫描, 并在样品下方逐点收集透射光. 该 方法使样品能够与局域在点源附近的衰逝波发生相 互作用进而突破分辨率极限, 然而当时的技术条件 无法满足此设想对光源强度、孔径尺寸、距离控制的 严格要求.

直到 1959年, Frait ${ }^{[31]}$ 成功开发了第一台铁磁共振 微波显微镜, Synge的想法才得以实现. 1962年, Soohoo ${ }^{[32]}$ 也独立地完成了类似的实验. 1972年, Ash 和Nicholls ${ }^{[33]}$ 利用准光学半球谐振腔完成了第一个真 正意义上的近场微波实验, 他们对 Synge的设计进行 了优化, 实现了在固定频率下小孔-样品间距的调制 和反射信号的相敏检测, 进一步提高了分辨率. 但是 早期的实验更多使用带孔谐振腔或雉形波导, 仪器 往往工作在其截止频率以下, 这会导致电磁波的严 重衰减 ${ }^{[32]}$. 为了解决此问题, Bryant和 Gunn ${ }^{[34]}$ 在 1965 年首次使用渐细同轴传输线的开端来探测材料的局 域电阻; 之后, Fee和 $\mathrm{Chu}^{[35]}$ 利用微同轴传输线作为探 针实现了微波和红外波段的物质表征, 灵敏度得到 了较大改善, 这也是首个用近场显微术的语言来描 述实验结果的工作.

20世纪80年代STM和AFM的发明标志着原子级 别距离控制的成功实现, 这进一步促进了 NMM的发 展. 之后便陆续出现了如衰逝微波探针显微镜 (evanescent microwave probe, EMP) $^{[36]}$ 、微波阻抗显微 镜(microwave impedance microscope, MIM) ${ }^{[37]}$ 等设备, 这些各具优势的 NMM在许多领域都得到了广泛应 用。

NMM测量原理大致如下: 微波源发射微波到探 针, 探针附近的电磁场在近场范围内与样品发生相 互作用, 仪器通过探测带有样品信息的调制波来重
构物性的分布. 因此, NMM的关键组成部件有近场 微波探针、距离控制元件、微波产生与探测电路.

NMM探针的主要作用是发射微波并探测信号, 其设计直接影响仪器的分辨率、灵敏度等重要性能指 标, 是NMM最重要的部件. 如图2所示, 目前较为常 用的探针结构有带孔波导管或谐振腔 ${ }^{[31]}$ 、同轴线或 同轴腔 ${ }^{[36,38]}$ 、平行线、AFM悬臂梁 ${ }^{[37] 、}$ 、磁线圈 ${ }^{[39]}$ 等. 其中, 图2(b)与 (d)相比, 前者在传输线的开端具有场 增强特征(STM针尖), 这一特征可以显著缩小场与样 品的作用范围从而得到约为针尖曲率半径的空间分 辨率.

精密控距的重要性表现在两个方面：一是为保 证足够高的分辨率，探针-样品间距(简称“间距”)须 在近场范围内; 二是由于样品表面通常不够平整而 测量结果又明显依赖于间距，为了保证测量结果的 可信度, 有必要通过精密控距方法来确保在空间扫 描时间距不变.

NMM常用的距离测控方法有STM测控 ${ }^{[40]}$ AFM 测控 $^{[37]}$ 、切变力测控 ${ }^{[41]}$ 等. STM利用样品隧穿电流强

(a)
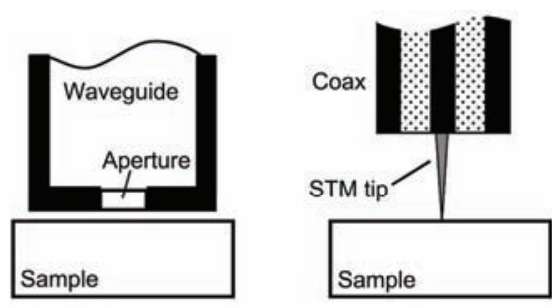

(b)

(c)
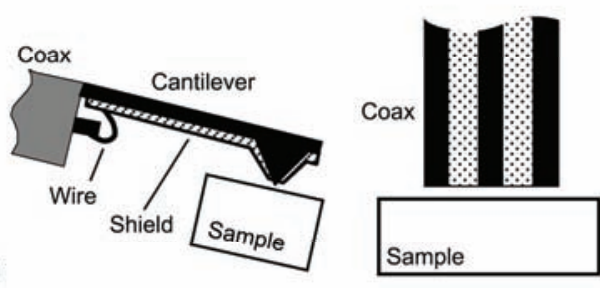

(d)

(e)
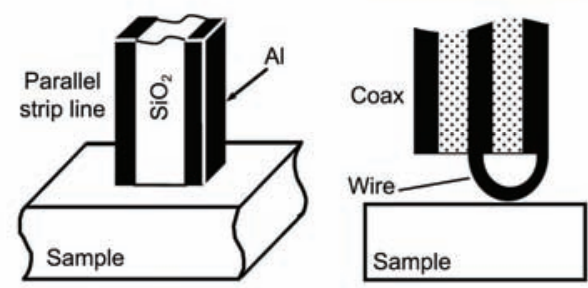

(f)

图 2 常用的NMM探针. (a) 带孔波导管; (b) STM针尖; (c) AFM针 尖; (d) 同轴线开口端; (e) 平行带状传输线; (f) 磁线圈 ${ }^{[27]}$

Figure 2 The main types of near-field microwave probes. (a) Aperture in a waveguide; (b) STM tip; (c) AFM tip; (d) open end of coaxial line; (e) parallel strip transmission line; (f) magnetic loop ${ }^{[27]}$ 
度的变化来控制间距，精度较高但适用范围局限于 导电材料; AFM悬臂梁会因样品、针尖原子之间的相 互作用力而发生位移, 实验中利用激光束的反射来 探测此位移进而控制间距; 在切变力测控技术中, 探 针沿平行于样品表面方向以其共振频率振动. 当间 距较小时, 原子力将对针尖施以横向切变力, 针尖的 振动幅度会因切变力阻尼而减小, 此振动幅度可以 用来控制间距. 该技术可以利用压电材料或者石英 音叉来实现.

NMM的另一重要组成部分是传输微波信号的电 路系统, 针对不同结构的探针及其相应的探测原理 有不同的设计, 但目的都是为了获取反映材料物性 的参数.

对于EMP, 微波由数字微波源产生并被分成两 路, 一路经耦合环进人同轴腔, 再由针尖发射到样品 处, 样品通过吸收、反射过程调制针尖附近的电磁场, 从而引起谐振腔共振频率 $f$ 和品质因数 $Q$ 的变化; 另 一路则进人移相器, 相位移动 $90^{\circ}$. 之后, 两路信号 在鉴相器中混频并进行相位比较, 从而得到参数的 变化 $\Delta f, \Delta Q^{[42]}$. 对于 MIM, 微波源产生的信号通过定 向耦合器传人针尖, 部分被样品表面反射回耦合器, 同时输人背景抑制信号, 之后两个信号被解调为与 针尖样品间阻抗有关的直流信号 ${ }^{[37]}$. 之后又出现了 AFM 与矢量网络分析仪 (vector network analyzer, VNA)结合的方式 ${ }^{[43]}$, VNA既作为微波源又作为探测 器, 它可以直接测量微波 $S$ 参数 ${ }^{[44]}$.

一般情况下, 探测电路直接得到的都是诸如共 振频率 $f$ 、品质因数 $Q 、 S$ 参数等微波系统参数, 因此 还需建立模型将所得参数与材料的物理性质联系起 来. 对于NMM, 一般采用集总元件模型、腔微扰理论 等方法分析微波参数与材料物性的关系.

对于非共振型的测量系统, 一般采用集总元件 模型 ${ }^{[55]}$. 在近场范围内, 探针和样品的相互作用可 等效为阻抗 $Z=R+\mathrm{i} X^{[27]}$, 其中电阻 $R$ 代表样品或探针 对电磁场能量的耗散, 电抗 $X$ 则针对低损耗材料即没 有实功率进人样品的情况. 根据玻印廷理论 ${ }^{[46]}$, 有

$$
\begin{gathered}
R=\frac{\omega}{\left|I_{i}\right|^{2}} \int_{V}\left(\frac{\sigma}{\omega}|\boldsymbol{E}|^{2}+\varepsilon_{0} \varepsilon^{\prime \prime}|\boldsymbol{E}|^{2}+\mu_{0} \mu^{\prime \prime}|\boldsymbol{H}|^{2}\right) \mathrm{d}^{3} x, \\
X=\frac{\omega}{\left|I_{i}\right|^{2}} \int_{V}\left(\boldsymbol{B} \cdot \boldsymbol{H}^{*}-\boldsymbol{E} \cdot \boldsymbol{D}^{*}\right) \mathrm{d}^{3} x,
\end{gathered}
$$

由(1)和(2)式可知阻抗 $Z$ 与材料的电导率、介电常数、 磁导率有关.
共振型的NMM则更多地采用腔微扰理论 ${ }^{[42]}$. 当 样品置于探针下方时, 二者的相互作用必然会对谐 振腔产生扰动, 这种扰动将导致腔体参数 $f, Q$ 发生变 化. 根据微扰理论 ${ }^{[47]}$, 有

$$
\begin{gathered}
\frac{\Delta f}{f}=-\frac{\int_{v}\left(\Delta \varepsilon \boldsymbol{E}_{1} \cdot \boldsymbol{E}_{0}+\Delta \mu \boldsymbol{H}_{1} \cdot \boldsymbol{H}_{0}\right) \mathrm{d} v}{\int_{v}\left(\varepsilon_{0} E_{0}^{2}+\mu_{0} H_{0}^{2}\right) \mathrm{d} v}, \\
\Delta \frac{1}{Q}=\frac{\int_{v}\left(\Delta \varepsilon^{\prime \prime} \boldsymbol{E}_{1} \cdot \boldsymbol{E}_{0}+\Delta \mu^{\prime \prime} \boldsymbol{H}_{1} \cdot \boldsymbol{H}_{0}\right) \mathrm{d} v}{\int_{v}\left(\varepsilon_{0} E_{0}^{2}+\mu_{0} H_{0}^{2}\right) \mathrm{d} v},
\end{gathered}
$$

容易看出 $f, Q$ 与材料的复介电常数、磁导率有关. 对 于此共振型的微扰系统, 一般有经验公式 $\Delta f \propto \Delta X_{s}{ }^{[48]}$, 再结合微波表面阻抗法的原理, 便可 以从谐振腔 $f$ 的变化得到超导穿透深度的信息.

由上述分析理论可以看出, 微波参数可通过 $\mathrm{NMM}$ 直接测量, 但若想定量地获取材料性质, 场的 求解也是必不可少的. 而相互作用范围内电磁场的 分布则需根据具体的探针结构来确定, 接下来本文 将具体介绍EMP的仪器结构及相应的定量分析理论.

\section{3 衰逝微波探针显微镜}

$\mathrm{NMM}$ 的分辨率主要由探针的特征尺寸决定, 过 小的特征尺寸虽能提升分辨率，但也会导致微波的 显著衰减, 而尺寸太大则会引人远场成分, 增加定量 分析的困难 ${ }^{[41]}$. 为了解决这些困难, Wei等人 ${ }^{[36]}$ 于 1996年提出EMP的设计, 并成功得到了样品表面水 平方向 $100 \mathrm{~nm}$ 的分辨率和 $10^{-4}$ 的灵敏度 ${ }^{[49]}$.

\subsection{EMP的结构}

如图3所示, EMP探针采用的是一个高品质因数 的1/4波长同轴谐振腔，腔闭端处的两个耦合环将同 轴腔与电路系统相连，分别起到发射和探测微波的 作用. 腔内中心导体尖化并与金属针相连，金属针从 腔体开端的小孔伸出至样品附近. 小孔由表面镀银 的白宝石构成，且小孔的尺寸不宜过大，以针尖恰好 穿过为宜, 这些做法既是为了屏蔽远场成分, 也是为 了降低针尖处微波的损耗, 保证较高的品质因数.

图4所示为EMP所用的切变力测控方法, 石英音 叉通过绝缘胶与金属针相连. 在EMP的实际测量中, 当某一材料处于与针尖的相互作用范围内，同轴腔 的共振频率 $f$ 与间距存在确定的关系, 因此可以通过 


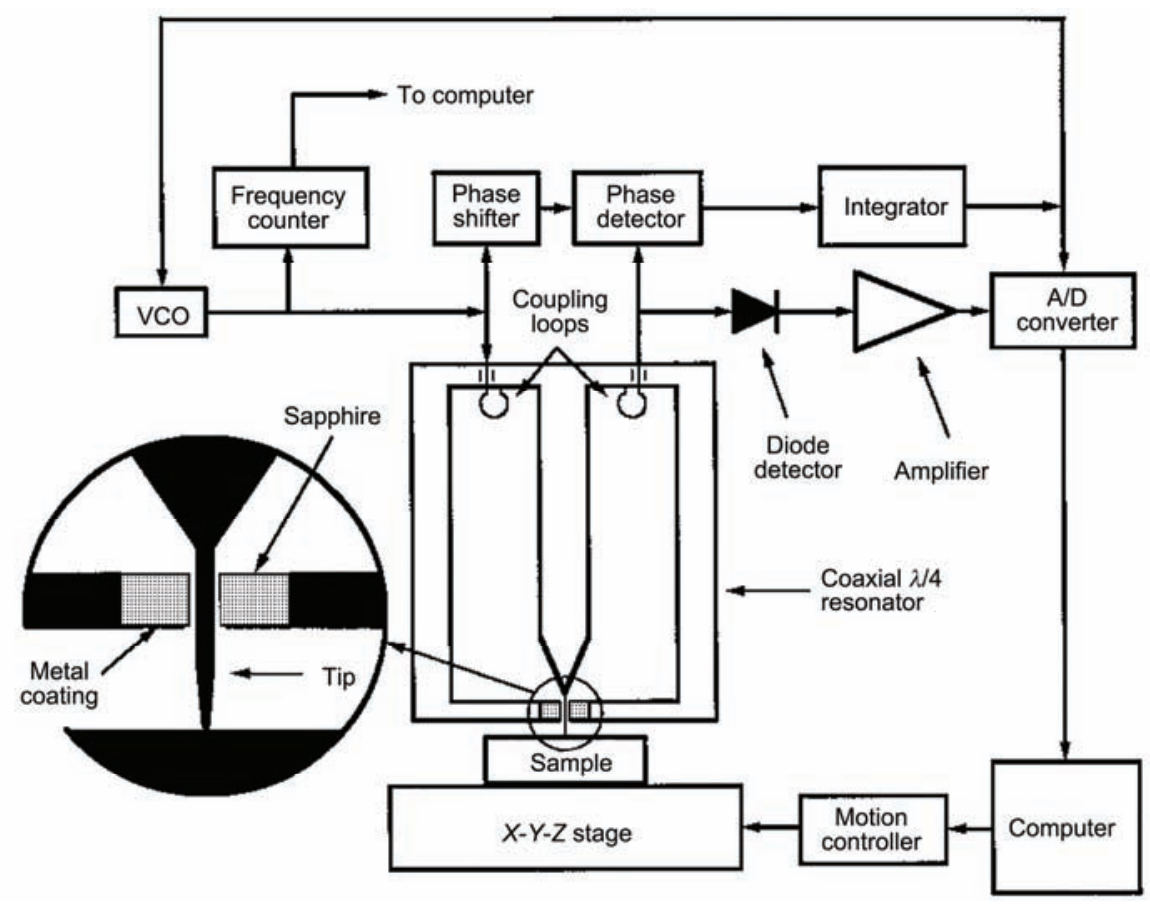

图 3 EMP结构图 ${ }^{[42]}$

Figure 3 The experimental configuration for the $\mathrm{EMP}^{[42]}$

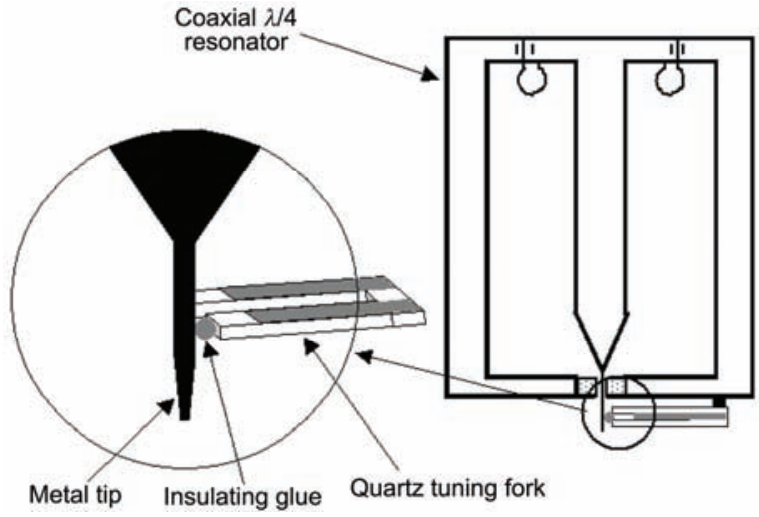

图 4 EMP常用的 $\mathrm{AFM}^{[41]}$

Figure 4 AFM incorporated in $\mathrm{EMP}^{[41]}$

锁定频率来控制距离 ${ }^{[50]}$, 这为EMP提供了一种新的 扫描模式一一等频模式. 在此模式下, 通过记录同轴 腔的品质因数来获得导电材料的电导率、表面阻抗等 信息.

\subsection{EMP的定量分析理论}

EMP属于共振型系统, 可以使用腔微扰法进行 分析. 根据前面的分析, 为了通过 $f, Q$ 得到样品的介 电常数等信息, 还需求得场的分布. 在近场范围内,
针尖与样品间的相互作用尺度较小且十分复杂, 一 般需要采用有限元分析(finite element analysis, FEA) 等数值方法. 但对于曲率半径较小的针尖, 其附近的 微波衰减极快, FEA方法并不适用 ${ }^{[51]}$.

针对这一情况, Gao等人 ${ }^{[42]}$ 于 1997 年提出了适用 于EMP的定量显微理论. 该理论采用了两个重要的 近似：近场微波是“准静态”的; 针尖可视为带电金属 微球. 对体材料, Gao等人采用如图 5所示的“镜像电 荷法”得到了样品中电场分布的解析式

$$
E_{1}=\frac{q}{2 \pi\left(\varepsilon+\varepsilon_{0}\right)} \sum_{n=1}^{\infty} \frac{b^{n-1}}{n} \frac{r \boldsymbol{e}_{r}+\left(z+\frac{R_{0}}{n}\right) \boldsymbol{e}_{z}}{\left[r^{2}+\left(z+\frac{R_{0}}{n}\right)^{2}\right]^{3 / 2}},
$$

并根据腔微扰理论进一步得到了 $\Delta f, \Delta Q$ 与材料介电 常数、介电损耗的关系 ${ }^{[42,49,52]}$

$$
\begin{gathered}
\frac{\Delta f}{f_{0}}=-A\left[\frac{\ln (1-b)}{b}+1\right], \\
\Delta\left(\frac{1}{Q}\right)=-\left(B_{Q}+B_{Q}{ }^{\prime} \tan \delta\right) \frac{\Delta f}{f},
\end{gathered}
$$

上式在接触模式下推得, 其中 $b=\left(\varepsilon-\varepsilon_{0}\right) /\left(\varepsilon+\varepsilon_{0}\right)$, $\tan \delta$ 为损耗角正切值, 系数 $A, B_{Q}, B_{Q}^{\prime}$ 由针尖、谐振腔 


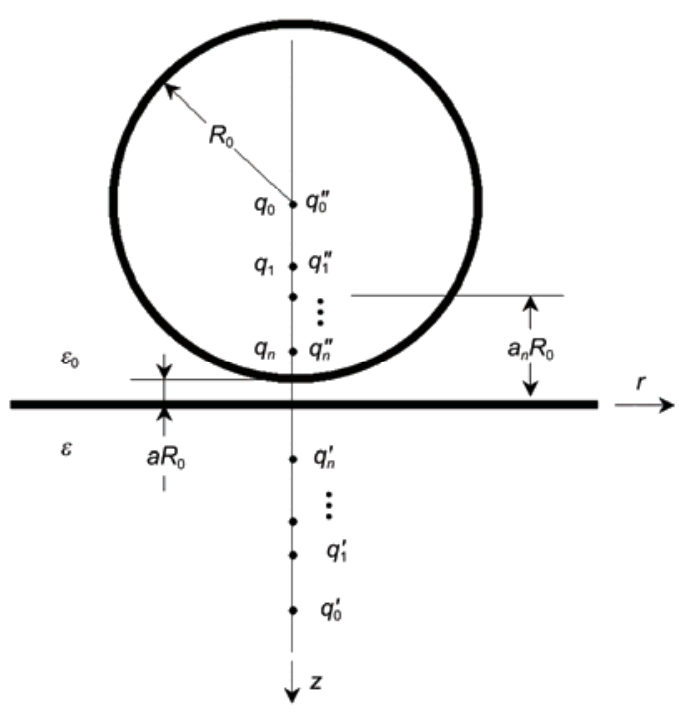

图 5 体材料的镜像电荷法 ${ }^{[41]}$

Figure 5 The iterative image charges in tip-bulk sample system ${ }^{[41]}$

几何形状决定, 可由标准样品测定. 随后, Gao等 人 $^{[51]}$ 又针对薄膜样品, 提出了无穷迭代的“树状”镜 像电荷法, 测得了十分精确的结果.

\subsection{EMP在超导研究中的应用}

随着定量理论的不断完善, EMP作为新型扫描近
场微波显微镜很快在超导表征和材料高通量篮选中 得到了应用 ${ }^{[41,53]}$, 下面举两个典型的例子.

高温超导材料 $\mathrm{YBa}_{2} \mathrm{Cu}_{3} \mathrm{O}_{7-x}(\mathrm{YBCO})$ 的发现首次将 超导转变温度提升到液氮温区, 其因极低的微波损耗 和较高的临界电流密度而被广泛应用于微波器件、医 学检测、电力传输等众多领域 ${ }^{[54]}$. 在 $\mathrm{YBCO}$ 材料中, 氧 含量的不均匀会导致不同区域超导转变温度 $T_{\mathrm{c}}$ 的差 异，这种不均匀性可由样品的表面电阻来表征.

Takeuchi等人 ${ }^{[54]}$ 采用湿刻技术得到了厚度为 300 $\mathrm{nm}$ 的 $\mathrm{YBCO}$ 薄膜方块 $(100 \mu \mathrm{m} \times 100 \mu \mathrm{m})$ 阵列, 为避免 材料与针尖的直接接触, 在样品上方沉积了 $200 \mathrm{~nm}$ 厚的 $\mathrm{SiO}_{2}$. 他们利用有低温系统的EMP对样品的局 域表面电阻进行表征, 结果如图6所示. 图6(a)为固 定点处 $f, Q$ 随温度的变化曲线, 由于频率变化较小, 可依据 $\Delta(1 / Q)$ 得到图 6(b) 所示的表面电阻的变化趋 势, 进而得出材料的相变温度大致为 80 90 K. 图 6(c)和(d)分别为室温和 $80 \mathrm{~K}$ 下的扫描图, 由此可见材 料边缘处的表面电阻比中间区域大，即边缘部分超 导相变不完全, 这很可能是由氧含量不均匀(刻蚀所 致)造成的 ${ }^{[29]}$.

高温超导微波器件通常需要高介电常数、低损耗 的材料作为衬底或中间层 ${ }^{[41]}$, 故利用组合薄膜技术

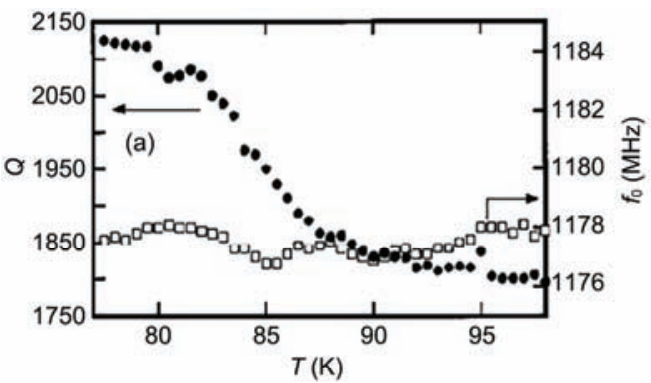

(c)

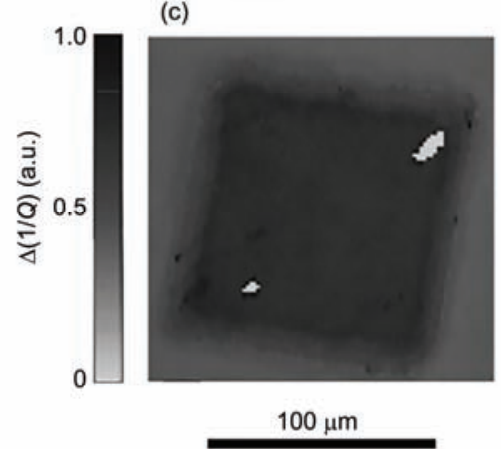

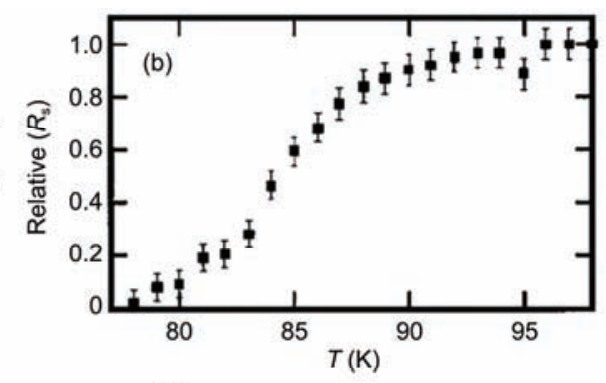

(d)

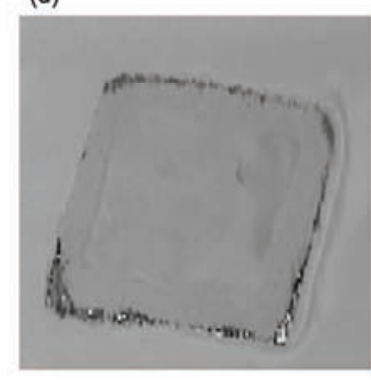

$100 \mu \mathrm{m}$

图 6 YBCO的EMP扫描结果. (a) $f, Q$ 随温度的变化; (b) 表面电阻随温度的变化; (c) 室温下 $\Delta(1 / Q)$ 扫描图; (d) $80 \mathrm{~K}$ 下 $\Delta(1 / Q)$ 扫描图 ${ }^{[54]}$

Figure 6 Data from EMP with the tip on YBCO. (a) Change of $f / Q$ with temperature; (b) relative surface resistance as a function of temperature; (c) $\Delta(1 / Q)$ images at room temperature; (d) $\Delta(1 / Q)$ images at $80 \mathrm{~K}^{[54]}$ 
寻找合适的介电材料是高温超导微波应用领域一项 重要的研究课题. $\mathrm{Ba}_{x} \mathrm{Sr}_{1-x} \mathrm{TiO}_{3}$ (BSTO)材料与大多数 铜氧化物超导体具有较好的晶格匹配, 同时其本身 具有较高的介电常数, 是一种富有潜力的材料. 然而 其介电损耗较高, 因此通过掺杂改善其损耗一直受 到广泛重视. Chang等人 ${ }^{[55]}$ 利用四元掩膜板技术, 在 1 平方英寸的 $\mathrm{LaAlO}_{3}(100)$ 单晶衬底上沉积出 256个不 同掺杂的 $\mathrm{Ba}_{x} \mathrm{Sr}_{1-x} \mathrm{TiO}_{3}(x=1.0,0.8,0.7$ 和0.5)样本, 如 图7(a)所示. 之后通过EMP对样品库进行快速表征, 分别测得每个掺杂区域的介电常数和介电损耗. 图 7(b)为所测得的介电常数对比图, 其中右上角 16 个小 样本掺杂了W元素, 比不掺杂的 1 号小样本颜色深, 这表明W元素的掺杂降低了BSTO的介电常数; 图 7(c)为所测得的介电损耗对比图, 其中W掺杂区域与
1号相比颜色较浅, 这说明W元素的掺杂同时降低了 损耗. W掺杂的样品虽然介电常数有所降低, 但介电 损耗的显著改善使该材料具有很高的应用价值.

Chang等人 ${ }^{[55]}$ 在实验中还合成了一些大尺寸薄 膜样品, 在表面光刻出叉指电极, 并测量了 $1 \mathrm{MHz}$ 的介电常数, 其结果与样本库的介电性质变化趋势 一致, 进一步说明了高通量合成与表征的可信度.

\section{4 总结与展望}

寻找具有更高转变温度的超导材料、探究超导微 观机制一直是凝聚态领域的重要课题. 近场微波显微 镜用于材料微波物性的高通量表征，具有无损、快速、 高分辨的巨大优势，在材料基因组计划中将起到非常 重要的作用 ${ }^{[56]}$. 它可以用于表征超导、介电 ${ }^{[29,57,58]}$ 、
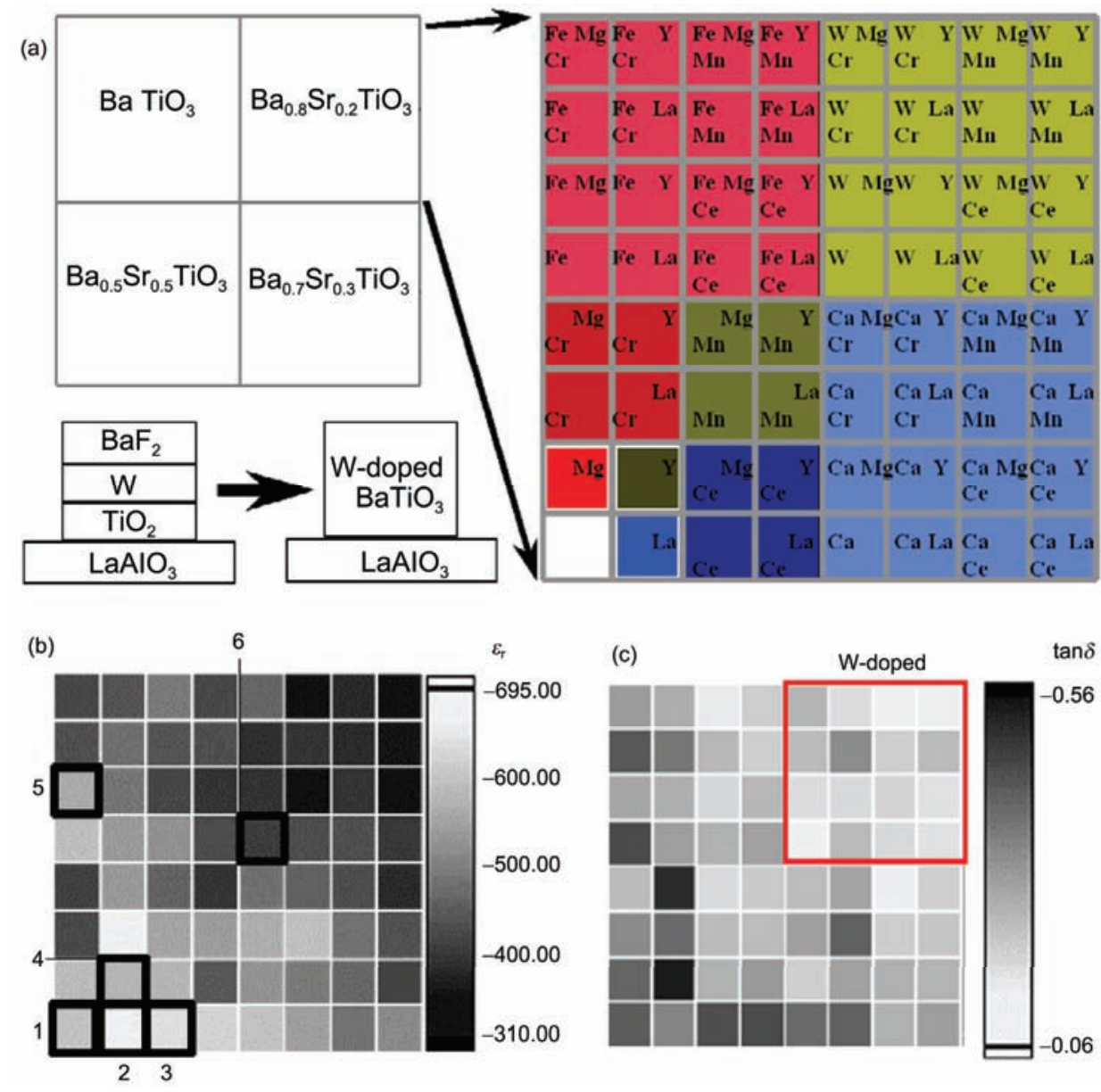

图 7 (网络版彩色)样本库的合成和表征结果. (a) 掺杂BSTO薄膜样本库合成原理图; (b) 样本库介电常数扫描图; (c) 损耗角的正切值扫描 图 $^{[41]}$

Figure 7 (Color online) Synthesis and characterization of the library. (a) Synthesis schematic of the doped BSTO library; (b) relative dielectric constant images; (c) loss tangent images ${ }^{[41]}$ 


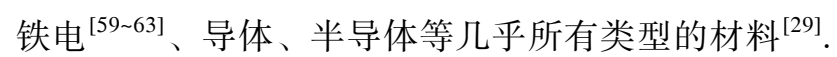
随着技术和理论的不断成熟, NMM在低维量子输 运 $^{[64,65]}$ 等新兴凝聚态领域也开始发挥作用, 利用低 温NMM研究金属绝缘体转变 ${ }^{[66,67]}$ 和相分离 ${ }^{[68,69]}$ 的工
作也相继被报道. 此外，由于材料的微波响应可以直 接反映低能准粒子动力学特性, NMM有望在噟能隙、 浴旋态等更多与超导材料相关的研究中发挥更大的 作用 ${ }^{[70]}$.

感谢中国科学技术大学高琛教授在仪器运行方面给予的指导和帮助, 感谢中国科学院物理研究所柳学榕研究员 和郑东宁研究员对文章成文的有益建议.

\section{参考文献}

1 Sun G F, Wong K W, Xu B R, et al. $T_{\mathrm{c}}$ enhancement of $\mathrm{HgBa}_{2} \mathrm{Ca}_{2} \mathrm{Cu}_{3} \mathrm{O}_{8+\delta}$ by Tl substitution. Phys Lett A, 1994, 192: 122-124

2 Scalapino D J. A common thread: The pairing interaction for unconventional superconductors. Rev Mod Phys, 2012, 84: 1383-1417

3 Jiang W, Peng J L, Li Z Y, et al. Transport properties of $\mathrm{Nd}_{1.85} \mathrm{Ce}_{0.15} \mathrm{CuO}_{4+\delta}$ crystals before and after reduction. Phys Rev B, 1993, 47: $8151-8155$

4 Wang H Z, Wang H, Ding H, et al. Progress in high-throughput materials synthesis and characterization (in Chinese). Sci Tech Rev, 2015, 33: 31-49 [王海舟，汪洪，丁洪，等. 材料的高通量制备与表征技术. 科技导报, 2015, 33: 31-49]

5 Wang X L, Xiao R J, Li H, et al. Oxysulfide LiAlSO: A lithium superionic conductor from first principles. Phys Rev Lett, 2017, 118: 195901

6 Shi Y J, Zhang X, Qin L, et al. Rapid preparations of $\mathrm{Bi}_{1-x} \mathrm{La}_{x} \mathrm{FeO}_{3 \pm \delta}$ thin films and their ferroelectric properties (in Chinese). Acta Phys

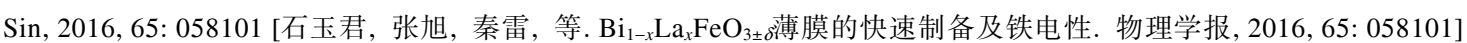

7 Yu H S, Yuan J, Zhu B Y, et al. Manipulating composition gradient in cuprate superconducting thin films. Sci China-Phys Mech Astron, 2017, 60: 087421

8 Koinuma H, Takeuchi I. Combinatorial solid-state chemistry of inorganic materials. Nat Mater, 2004, 3: 429-438

9 Kennedy K, Stefansky T, Davy G, et al. Rapid method for determining ternary-alloy phase diagrams. J Appl Phys, 1965, 36: 3808-3810

10 Xiang X D, Sun X, Briceho G, et al. A combinatorial approach to materials discovery. Science, 1995, 268: 1738-1740

11 Gao C, Bao J, Luo Z L, et al. Recent progresses in the combinatorial materials science (in Chinese). Acta Phys-Chim Sin, 2006, 22: 899-912 [高琛, 鲍骏, 罗震林, 等. 组合材料学研究进展. 物理化学学报, 2006, 22: 899-912]

12 Mao S S. High throughput growth and characterization of thin film materials. J Cryst Growth, 2013, 379: 123-130

13 Xu R, Zheng Z Y, Ji W, et al. Advanced scanning microwave microscopy (in Chinese). Prog Phys, 2015, 35: 241-256 [许瑞, 郑志月, 季威, 等. 新型扫描微波显微术. 物理学进展, 2015, 35: 241-256]

14 Onnes H K. The resistance of pure mercury at helium temperatures. Comm Phys Lab Univ Leiden, 1911, 12: 120b

15 Meissner W, Ochsenfeld R. A new effect in onset of superconductivity. Naturwiss, 1933, 21: 787-788

16 London F, London H. The electromagnetic equations of the supraconductor. Proc Roy Soc A, 1935, 149: 71-88

17 Zehetmayer M. A review of two-band superconductivity: Materials and effects on the thermodynamic and reversible mixed-state properties. Supercon Sci Tech, 2013, 26: 043001

18 Armitage N P, Fournier P, Greene R L. Progress and perspectives on electron-doped cuprates. Rev Mod Phys, 2010, 82: 2421-2487

19 Zhang Q R, Xu Z A, Wang J S, et al. Characteristic parameters $\lambda, \xi$ and $\Delta$ of high $T_{\mathrm{c}}$ oxide superconductors (in Chinese). Prog Phys, 1992，12：436-479 [张其瑞, 许祝安, 王劲松, 等. 高临界温度氧化物超导体的超导特征参量： $\lambda, \xi$ 和 $\Delta$. 物理学进展, 1992, 12: 436-479]

20 Zhang Q R. High Temperature Superconductivity (in Chinese). Hangzhou: Zhejiang University Press, 1992 [张其瑞. 高温超导电性. 杭 州: 浙江大学出版社, 1992]

21 Drabeck L, Grüner G, Chang J J, et al. Millimeter-wave surface impedance of $\mathrm{YBa}_{2} \mathrm{Cu}_{3} \mathrm{O}_{7}$ thin films. Phys Rev B, 1989, 40: 7350-7353

22 Wu D H, Mao J, Mao S N, et al. Temperature dependence of penetration depth and surface resistance of $\mathrm{Nd}_{1.85} \mathrm{Ce}_{0.15} \mathrm{CuO}_{4}$. Phys $\mathrm{Rev} \mathrm{Lett}$, 1993, 70: 85-88

23 Hardy W N, Bonn D A, Morgan D C, et al. Precision measurements of the temperature dependence of $\lambda$ in $\mathrm{YBa}_{2} \mathrm{Cu}_{3} \mathrm{O}_{6.95}: \mathrm{Strong}$ evidence for nodes in the gap function. Phys Rev Lett, 1993, 70: 3999-4002

24 Jia Y L, Yang H, Yuan J, et al. A brief analysis of annealing process for electron-doped cuprate superconductors (in Chinese). Acta Phys Sin, 2015, 64: 217401 [贾艳丽，杨桦，袁洁，等. 浅析电子型掺杂铜氧化物超导体的退火过程. 物理学报, 2015, 64: 217401] 
Yuan J, He G, Yang H, et al. Research trends in electron-doped cuprate superconductors. Sci China-Phys Mech Astron, 2015, 58: 107401

Zhang X, Yu H S, He G, et al. Transport anomalies and quantum criticality in electron-doped cuprate superconductors. Phys C, 2016, 525-526: 18-43

Anlage S M, Talanov V V, Schwartz A R. Scanning Probe Microscopy: Electrical and Electromechanical Phenomena at the Nanoscale. New York: Springer, 2007

Xiang X D, Gao C. Quantitative complex electrical impedance microscopy by scanning evanescent microwave microscope. Mater Charact, 2002, 48: 117-125

Gao C, Xiang X D, Wu Z Q. Novel scanning tip microwave near-field microscopy (in Chinese). Physics, 1999, 28: 630-634 [高琛, 项晓 东，吴自勤. 新型扫描近场微波显微术. 物理, 1999, 28: 630-634]

Synge E H. A suggested method for extending microscopic resolution into the ultra-microscopic region. Philos Mag, 1928, 6: 356-362

Frait Z. The use of high-frequency modulation in studying ferromagnetic resonance. Czechosl Journ Phys, 1959, 9: 403-404

Soohoo R F. A microwave magnetic microscope. J Appl Phys, 1962, 33: 1276-1277

Ash E A, Nicholls G. Super-resolution aperture scanning microscope. Nature, 1972, 237: 510-512

Bryant C A, Gunn J B. Noncontact technique for the local measurement of semiconductor resistivity. Rev Sci Instrum, 1965, 36: 1614-1617

Fee M, Chu S. Scanning electromagnetic transmission line microscope with sub-wavelength resolution. Opt Commun, 1989, 69: 219-224 Wei T, Xiang X D, Wallace-Freedman W G, et al. Scanning tip microwave near-field microscope. Appl Phys Lett, 1996, 68: 3506-3508

Lai K, Ji M B, Leindecker N, et al. Atomic-force-microscope-compatible near-field scanning microwave microscope with separated excitation and sensing probes. Rev Sci Instrum, 2007, 78: 063702

Tabib-Azar M, Su D P, Pohar A, et al. $0.4 \mu \mathrm{m}$ spatial resolution with $1 \mathrm{GHz}(\lambda=30 \mathrm{~cm})$ evanescent microwave probe. Rev Sci Instrum, 1999, 70: 1725-1729

Lee S C, Vlahacos C P, Feenstra B J, et al. Magnetic permeability imaging of metals with a scanning near-field microwave microscope. Appl Phys Lett, 2000, 77: 4404-4406

Lee J, Long C J, Yang H, et al. Atomic resolution imaging at $2.5 \mathrm{GHz}$ using near-field microwave microscopy. Appl Phys Lett, 2010, 97: 183111

Gao C, Hu B, Takeuchi I, et al. Quantitative scanning evanescent microwave microscopy and its applications in characterization of functional materials libraries. Meas Sci Tech, 2005, 16: 248-260

Gao C, Wei T, Duewer F, et al. High spatial resolution quantitative microwave impedance microscopy by a scanning tip microwave near-field microscope. Appl Phys Lett, 1997, 71: 1872-1874

Huber H P, Moertelmaier M, Wallis T M, et al. Calibrated nanoscale capacitance measurements using a scanning microwave microscope. Rev Sci Instrum, 2010, 81: 113701

Imtiaz A, Wallis T M, Kabos P. Near-field scanning microwave microscopy. IEEE Microw Mag, 2014, 15: 52-64

Lai K, Kundhikanjana W, Kelly M, et al. Modeling and characterization of a cantilever-based near-field scanning microwave impedance microscope. Rev Sci Instrum, 2008, 79: 063703

Jackson J D. Classical Electrodynamics. New York: Wiley, 1999

Pozar D M. Microwave Engineering. New York: Addison-Wesley, 1990

Maeda A, Kitano H, Inoue R. Microwave conductivities of high- $T_{\mathrm{c}}$ oxide superconductors and related materials. J Phys: Condens Matter, 2005, 17: R143-R185

Gao C, Xiang X D. Quantitative microwave near-field microscopy of dielectric properties. Rev Sci Instrum, 1998, 69: 3846-3851

Duewer F, Gao C, Takeuchi I, et al. Tip-sample distance feedback control in a scanning evanescent microwave microscope. Appl Phys Lett, 1999, 74: 2696-2698

51 Gao C, Hu B, Zhang P, et al. Quantitative microwave evanescent microscopy of dielectric thin films using a recursive image charge approach. Appl Phys Lett, 2004, 84: 4647-4649

Gao C, Duewer F, Xiang X D. Quantitative microwave evanescent microscopy. Appl Phys Lett, 1999, 75: 3005-3007

Xiang X D. Combinatorial materials synthesis and screening: An integrated materials chip approach to discovery and optimization of functional materials. Annu Rev Mater Sci, 1999, 29: 149-171

5 Takeuchi I, Wei T, Duewer F, et al. Low temperature scanning-tip microwave near-field microscopy of $\mathrm{YBa}_{2} \mathrm{Cu}_{3} \mathrm{O}_{7-x}$ films. Appl Phys Lett, 1997, 71: 2026-2028

Chang H, Gao C, Takeuchi I, et al. Combinatorial synthesis and high throughput evaluation of ferroelectric/dielectric thin-film libraries for microwave applications. Appl Phys Lett, 1998, 72: 2185-2187

6 Green M L, Takeuchi I, Hattrick-Simpers J R. Applications of high throughput (combinatorial) methodologies to electronic, magnetic, optical, and energy-related materials. J Appl Phys, 2013, 113: 231101 
57 Liu X R, Hu B, Liu W H, et al. The theoretical calibration coefficient in the measurement of nonlinear dielectric constant with a scanning tip microwave near-field microscopy (in Chinese). Acta Phys Sin, 2003, 52: 34-38 [柳学榕, 胡泊, 刘文汉, 等. 扫描近场微波显微镜 测量非线性介电常数的理论校准系数. 物理学报, 2003, 52: 34-38]

58 Gao C, Duewer F, Lu Y, et al. Quantitative nonlinear dielectric microscopy of periodically polarized ferroelectric domains. Appl Phys Lett, 1998, 73: 1146-1148

59 Seidel J, Martin L W, He Q, et al. Conduction at domain walls in oxide multiferroics. Nat Mater, 2009, 8: 229-234

60 Catalan G, Seidel J, Ramesh R, et al. Domain wall nanoelectronics. Rev Mod Phys, 2012, 84: 119-156

61 Ma E Y, Bryant B, Tokunaga Y, et al. Charge-order domain walls with enhanced conductivity in a layered manganite. Nat Commun, 2015, 6: 7595

62 Ma E Y, Cui Y T, Ueda K, et al. Mobile metallic domainwalls in an all-in-all-out magnetic insulator. Science, 2015, 350: 538-541

63 Tselev A, Yu P, Cao Y, et al. Microwave a.c. conductivity of domain walls in ferroelectric thin films. Nat Commun, 2016, 7: 11630

64 Cui Y T, Wen B, Ma E Y, et al. Unconventional correlation between quantum Hall transport quantization and bulk state filling in gated graphene devices. Phys Rev Lett, 2016, 117: 186601

65 Ma E Y, Calvo M R, Wang J, et al. Unexpected edge conduction in mercury telluride quantum wells under broken time-reversal symmetry. Nat Commun, 2015, 6: 7252

66 Hyun S, Cho J H, Kim A, et al. Coexistence of metallic and insulating phases in epitaxial $\mathrm{CaRuO}_{3}$ thin films observed by scanning microwave microscopy. Appl Phys Lett, 2002, 80: 1574-1576

67 Lai K, Nakamura M, Kundhikanjana W, et al. Mesoscopic percolating resistance network in a strained manganite thin film. Science, 2010, 329: 190-193

68 Takahashi H, Imai Y, Maeda A. Observation of mesoscopic phase separation in $\mathrm{K}_{x} \mathrm{Fe}_{y} \mathrm{Se}_{2}$ by scanning microwave microscopy. Phys C, 2015, 518: 33-35

69 Takahashi H, Imai Y, Maeda A. Near-field microwave imaging of inhomogeneous $\mathrm{K}_{x} \mathrm{Fe}_{y} \mathrm{Se}_{2}$ : Separation of topographic and electric features. Appl Phys Lett, 2015, 106: 233106

70 Takahashi H, Imai Y, Maeda A. Low-temperature-compatible tunneling-current-assisted scanning microwave microscope utilizing a rigid coaxial resonator. Rev Sci Instrum, 2016, 87: 063706 


\title{
Applications and perspective of near-field microwave microscope in high-throughput characterizations of superconducting materials
}

\author{
QIN MingYang, SHI YuJun, WEI ZhongXu, ZHU BeiYi, YUAN Jie* \& JIN Kui* \\ Beijing National Laboratory for Condensed Matter Physics, Institute of Physics, Chinese Academy of Sciences, Beijing 100190, China \\ * Corresponding authors, E-mail: yuanjie@iphy.ac.cn; kuijin@iphy.ac.cn
}

High- $T_{\mathrm{c}}$ superconductors are promising in applications of power transmission, magnetic levitation, magnetic resonance imaging and other fields which are limited so far by the low critical temperature of present materials, so searching for superconducting materials with higher $T_{\mathrm{c}}$ is of much significance. However, in the process of exploring new materials and establishing a complete phase diagram, a new route of high efficiency and accuracy is highly desired. Fortunately, the materials genome initiative (MGI) composed of calculations and data processing, high-throughput synthesis and fast screening technologies has been showing great advantages in shortening the $\mathrm{R} \& \mathrm{D}$ cycle and saving the cost. For example, in our recent work, the continuous-composition-spread $\mathrm{La}_{2-x} \mathrm{Ce}_{x} \mathrm{CuO}_{4}$ films synthesized by the third-generation combinatorial film technique enable us to be closer to the quantum critical point in cuprate superconductors and the $T_{\mathrm{c}}$-continuous-variation FeSe film is helpful to track the superconductivity in the iron-based family. As the indispensable experimental component, the high-throughput synthesis and screening techniques play the key role in the MGI. In this review, we briefly introduce the commonly used combinatorial film synthesizing technologies as well as several rapid characterization techniques such as micro-area X-ray diffractometer, imaging ellipsometer and laser thermal characterization techniques. The common features for the high-throughput characterization methods are pointed out that the "sharp tip" which can scan on the sample surface or the probe array is necessary. The spatial resolution of the facility is determined by the dimension of the tip or the resolution of the virtual "tip". According to these clues, near-field microwave microscope (NMM) of high resolution and nondestructive scanning, a typical high-throughput electromagnetic characterization method, is beneficial for characterizing combinatorial superconducting films since the surface impedance of superconductors is related to the magnetic penetration depth which can reflect the feature of superconducting gap. Thus, taking the developing history of NMM as a starting point, we summarize its working principle, instrumental configuration including near-field microwave probe, distance-control component, microwave generation and detection circuit, as well as commonly used analysis methods, such as lumped element model and cavity perturbation theory. Then the design concept and theory support of evanescent microwave probe (EMP) are explained in detail as an example of NMM. Two prototypes about superconductors of EMP studies are also demonstrated. Finally, some potential applications of NMM in the high-throughput characterization of combinatorial superconducting films have been prospected. We expect that the NMM will play a more important role in disclosing the nature of pseudo gap and the vortex dynamics.

materials genome initiative, high-throughput experimental technology, combinatorial film, near-field microwave microscope

doi: 10.1360/N972017-00722 